\title{
A NOTE ON THE SUFFICIENCY OF SOKOLOWSKI'S CRITERION FOR CONTEXT-FREE, LANGUAGES
}

\author{
Anton NIJHOLT \\ Faculty of Science, Department of Informatics, Nijmegen University, The Netherlands
}

Received 10 May 1982

Keywords: Context-free languages, pumping lemmas

Sokolowski [5] introduced a condition on languages which can be used to show that a language is not context-free. This condition is the following: Let $L \subseteq \Sigma^{*}$ be a context-free language. For every subset $\Sigma^{\prime}$ of $\Sigma$ containing at least two distinct letters and for every $u, v, w$ in $\Sigma^{*}$, if $\{u x v x w \mid x$ in $\left.\Sigma^{\prime+}\right\} \subseteq L$, then there exist two different words $x^{\prime}$, $x^{\prime \prime}$ in $\Sigma^{\prime+}$, such that $u x^{\prime} v x^{\prime \prime} w$ is in L. In [5] the non-context-free language

$L=\left\{x c^{k} x \mid k>0\right.$ and $\left.x \in\{a, b\}^{+}\right\} \cup\{a, b\}^{+}$

is used as an example to show that the "Classical Pumping Lernma" [1] cannot be used to determine that $L$ is non-context-free, while the above given criterion simply leads to the conclusion that $\mathrm{L}$ is not context-free.

In [5] the question is raised whether the criterion is not only a necessary, but also a sufficient one. The answer is negative and this can be understood with the help of a simple variation of $L$. Consider the language

$L^{\prime}=\left\{\operatorname{xcxx} \mid x \in\{a, b\}^{+}\right\} \cup\left\{x c x \mid x \in\{a, b\}^{+}\right\}$.

For $u=\varepsilon, v=c$ and $w=\varepsilon$ we have $\{x c x \mid x \in$ $\left.\{a, b\}^{+}\right\} \subseteq L^{\prime}$. However, there exist two different words $x$ and $x x$ such that $x c x x$ is also in $L^{\prime}$. Hence, the criterion is satisfied while $L^{\prime}$ is not coniext-free.

It is interesting to note that the Ogden-like languages (cf. [3]) of the form $B_{p}=A_{p} \cup$ $X^{*}\{a a, b b\} X^{*}$ with $X=\{a, b\}$ and $A_{p}=\left\{(a b)^{n} \mid n\right.$ $\in P\}$, where $P$ is a subset of the natural numbers, also satisfy Sokolowki's criterion. Notice that $P$ can be chosen in such a way that $A_{p}$ and therefore $B_{p}$ is not context-free, not context-sensitive, not recursive or not recursively enumerable. The languages $B_{p}$ illustrate that although Sokolowski's criterion is a very useful tool in determining noncontext-free-ness, it only considers one of the many conditions of a language being non-context-free.

\section{References}

[1] Y. Bar-Hillel, M. Perles and E. Shamir, On formal properties of simple phrase structure grammars, Zeitschr. Phonetik, Sprachwiss. und Kommunikationsforsch. 14 (1961) 143-172.

[2] L. Boasson, A remark on Ogden's lemma, EATCS-Bull. 4 (1978) 3-4.

[3] L. Boassnn and S. Horvath, On languages satisfying Ogden's lemma, RAIRO Inform. Theorique 12 (1978) 201-202.

[4] W. Ogden, A helpful result for proving inherent ambiguity, Math. Systems Theory 2 (1968) 191-197.

[5] S. Sokolowski, A method for proving programming languages non-context-free, Inform. Process. Lett. 7(3) (1978) 151-153.

[6] D.S. Wise, A strong pumping lemma for context-free languages, Theoret. Comput. Sci. 3 (1976) 359-369. 